Research Article

\title{
Epidemic Trajectory in the Absence of Non- Pharmacological Interventions in India: An Insight into Post Vaccine Introduction Phase
}

\author{
Aditya Athotra', Sudarshan Ramaswamy², Meera Dhuria ${ }^{3}$, Anil D Patil ${ }^{4}$, Jugal Kishore ${ }^{5}$, \\ SK Jain ${ }^{6}$, Sujeet Kumar Singh ${ }^{7}$ \\ ${ }^{1}$ Statistical Officer, ${ }^{4}$ Additional Director, Statistical Monitoring and Evaluation Cell, NCDC, Delhi, India. \\ ${ }^{2}$ Consultant-Health Emergencies, NOA, World Health Organisation (Currently Posted at NCDC, Delhi), India. \\ ${ }^{3}$ Deputy Director, ${ }^{6}$ Additional Director \& Head, Department of Epidemiology Division, NCDC, Delhi, India. \\ ${ }^{5}$ Director Professor and Head, Dept. Community Medicine, VMMC\& Safdarjung Hospital, Delhi, India. \\ ${ }^{7}$ Director, NCDC, Delhi, India.
}

DOI: https://doi.org/10.24321/2455.7048.202027

\section{I $\quad \mathbf{N} \quad \mathbf{F} \quad \mathbf{O}$}

\section{Corresponding Author:}

Meera Dhuria, Department of Epidemiology

Division, NCDC, Delhi, India.

E-mail Id:

meera.dhuria@gov.in

Orcid Id:

https://orcid.org/0000-0001-9053-6738

How to cite this article:

Athotra A, Ramaswamy S, Dhuria M, Patil AD, Kishore J, Jain SK et al. Epidemic Trajectory in the Absence of Non-Pharmacological Interventions in India: An Insight into Post Vaccine Introduction Phase. Epidem Int 2020; 5(4): 16-21.

Date of Submission: 2021-02-18

Date of Acceptance: 2021-03-12

\section{$\begin{array}{llllllll}\mathbf{A} & \mathbf{B} & \mathbf{S} & \mathbf{T} & \mathbf{R} & \mathbf{A} & \mathbf{C} & \mathbf{T}\end{array}$}

Background: Non-Pharmacological Interventions (NPIs) have proven to be effective in controlling and reducing the spread of SARS-CoV- 2 in the population. During the year 2020, before vaccine introduction, India has been through various phases of COVID-19 pandemic response such as nationwide lockdown phase 1 to 4 and unlock phases 1-8. Although India's vaccination program against COVID-19 has started, it is still in the initial phases and considering the humongous population of India, coverage of entire population with vaccine needs time.

Methodology: We designed a model showing the projections of expected incident cases of COVID-19 under two scenarios for the month of February 2021. In the first scenario, $R_{t}$ value expected to be observed during February 2021 if all the NPIs are removed was considered. In the second scenario, $R_{t}$ value projected as per the current trend with NPIs in place was considered. Model projections of both these scenarios were done for India and also for Delhi.

Result: Our simulation model quantifies the effect of Non-pharmacological interventions on the current pandemic situation in India and Delhi, which concludes that relaxation in preventive measures or COVID-19 appropriate behaviors or ceasing of NPIs shall see an exponential rise in the daily incident cases. Comparing the trajectories for India and Delhi, it can be deduced that if NPIs cease to exist for one month, the daily incident cases can be many times higher of normal in India and also in Delhi by the end of February 2021.

Conclusion: NPIs remain to play a major role in containing the spread and minimizing the effects of COVID-19 pandemic. Any kind of relaxation in NPIs can lead to sudden surge of incident cases and correspondingly may increase the death toll.

Keywords: COVID-19, SARS-CoV-2, COVID-19 Appropriate Behavior 


\section{Introduction}

It has been a year since the first official case of COVID-19 was identified (31 Dec 2020) in Wuhan, China. ${ }^{1}$ The causative organism nCoV (SARS CoV-2) ever since its inception, has spread across the whole world in the form of a pandemic and its effects have caused a widespread catastrophe throughout the globe. India witnessed its first case on 31 January 2020 and currently has witnessed a cumulative case count of 10,925,710 and 1,55,813 deaths as on 16 Feb 2020. ${ }^{2,3}$ India launched its COVID-19 vaccine drive in the country on 16 Jan $2021 .{ }^{4}$ On the first day, around 1.94 lakh beneficiaries were vaccinated. ${ }^{5}$ Ever since, India has been progressively covering its huge population (starting from frontline health workers to people $>50$ years of age and people $<50$ years with co-morbid conditions) ${ }^{6}$ under the COVID19 vaccination program. However, during the year 2020, before vaccine introduction, India has been through various phases of COVID-19 pandemic response such as nationwide lockdown phase 1 to 4 and unlock phases 1-8 (Figure 1). India's response to the pandemic include several strategies over the course of time such as increasing the testing capacity, upgrading healthcare infrastructure \& manpower, procurement of logistics, continuous epidemiological surveillance, scientific research on COVID-19 disease transmission, biological outcome, treatment \& vaccine production etc. Behavioural and social interventions also have played a great role in aiding efforts to mitigate the effects of this pandemic.

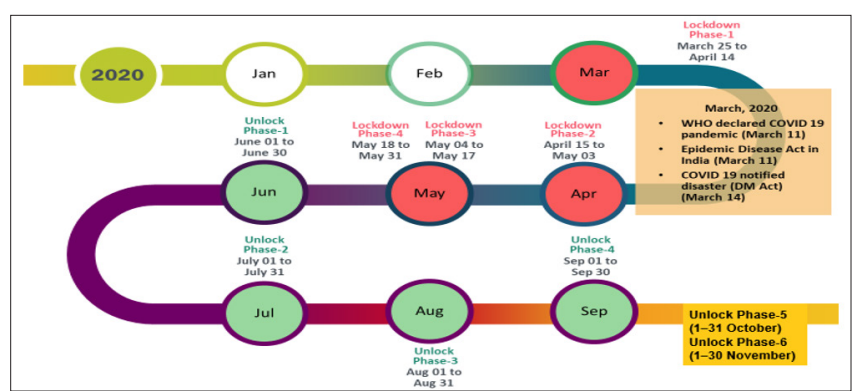

Figure I.Phases of COVID-19 pandemic response in India, 2020

Although India's vaccination program against COVID-19 has started, it is still in the initial phases and considering its huge population, coverage of entire population needs time. Effectiveness of vaccination program depends on many factors such as vaccination coverage, herd immunity threshold, efficacy of vaccine and the frequency of adverse effects following immunization. Until we reach the phase of elimination, it is imperative to continue adhering to preemptive non-pharmacological interventions for having the best cost-effectives. Non Pharmacological Interventions (NPIs) have proven to be effective in controlling and reducing the spread of SARS-CoV-2 in the population, which is quite evident from the actual epidemic trajectory of India as well (Figure 2). The current $R_{t}$ value (time dependent Reproduction Number- defined as the expected number of cases directly generated by one case in a population where all individuals are susceptible to infection) is less than 1 , which implies that the number of recoveries now outnumber the new infections.

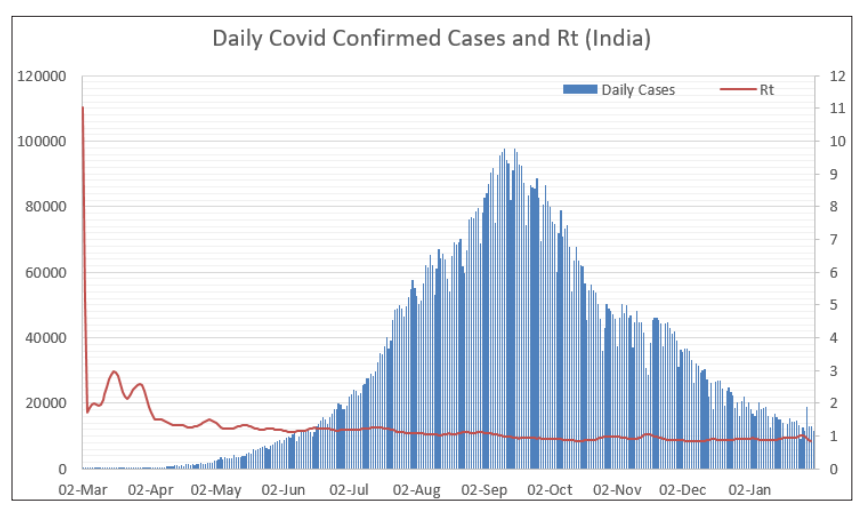

Figure 2.Daily incidence of COVID- 19 cases in India (Jan 30, 2020 to Jan 3I, 202 I) - The epidemic curve with the Rtvalue

Since the effect of vaccination is yet to be seen, this value of $R_{t}$ incorporates the effect of all the (NPIs) measures (including social distancing measures, masks, rapid identification of cases, closure of schools, travel restrictions etc.) taken by the Government of India. Interventional strategies for prevention and control heavily rely on intersectoral coordination/ collaboration, community engagement, community participation and ownership. Assuming that the reduction in $R_{t}$ is due to NPIs, we conducted a study with an aim to assess the epidemic scenario if India moves to a prepandemic state (terminate all the interventions initiated by the government, if population shall stop following COVID-19 appropriate behaviors) and all the other NPIs cease to exist immediately. Objective of the current study is to assess the benefits of continuing the NPIs against COVID-19 in future.

\section{Methodology}

In order to fulfill the objective of the study, we designed a model showing the projections of expected incident cases of COVID-19 under two scenarios for the month of February 2021. In the first scenario, $R_{t}$ value expected to be observed during February 2021 if all the NPIs are removed was considered. In the second scenario, $R_{t}$ value projected as per the current trend with NPIs in place was considered. Model projections of both these scenarios were done for India and also for Delhi. The period of interest for the required study is chosen from February 01, 2021 to March 01, 2021 and the latest data set of the period from January 01, 2021 to January 31, 2021 has been used for the simulation for the mentioned period. To provide more realistic and reliability to the model estimates, the most recent period prior to period of interest was chosen. 
After determining the $\mathrm{R}$ t-value and identifying the time period, following model has been designed to simulate plausible epidemic trajectories and project future incidence $(\lambda t)$. The model relies on a branching process where daily incidence follows a Poisson process determined by a daily infectiousness, computed as: ${ }^{8}$

$$
\lambda_{t}=\sum_{s=1}^{t-1} R s y_{s} w(\mathrm{t}-\mathrm{s})
$$

Where:

- $w(t-s)$ is the Probability Mass Function (PMF) of the serial interval

- $\quad(t-s)$ is a random variable representing serial interval (time between successive cases in a chain of transmission)

- ys is the incidence (by date of onset) at time " $s$ "

- $\mathrm{R}_{\mathrm{s}}$ is the effective reproduction number (average number of secondary cases by infected case) at time " $s$ "

Estimation of PMF: As per previous literature review, serial interval of mean 6 days and variance of 3.8 days has been assumed for the model. ${ }^{9}$ Gamma distribution has been assumed for estimating serial interval time as it is positively skewed. Based on these assumptions, Probability Mass Function (PMF) of the serial interval was calculated and a graphical representation is seen in Figure 3 . It is quite evident that the probability of transmitting the infection is higher on day 4, 5 and day 6 and as the time progress the probability is reducing.

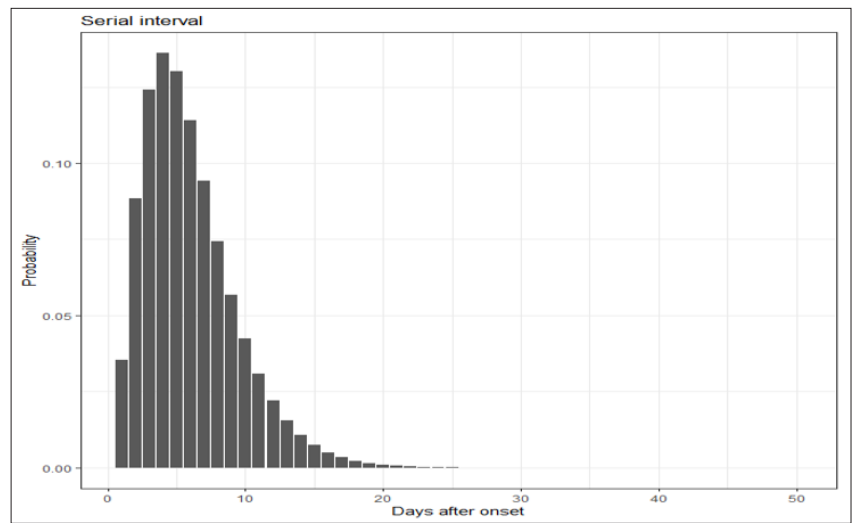

Figure 3.Serial interval distribution representing the probability of passing infections as the time progress

Estimation of Ys: We collected the daily incidence of COVID-19 cases of India and Delhi since the beginning of the pandemic. ${ }^{10} \mathrm{~A}$ graph of epidemic curve along with time dependent $R_{t}$ during the respective period was plotted for India (Figure 2) and Delhi (Figiure 4). Daily case incidence of COVID-19 respectively for India and Delhi on Jan 31, 2020 was considered as Ys.
Estimation of Rs: $R s$ is the $R_{t}$ value during the study period (February 2021) "s". $R_{t}$ is calculated based on the daily incident cases and the serial interval values. ${ }^{9}$

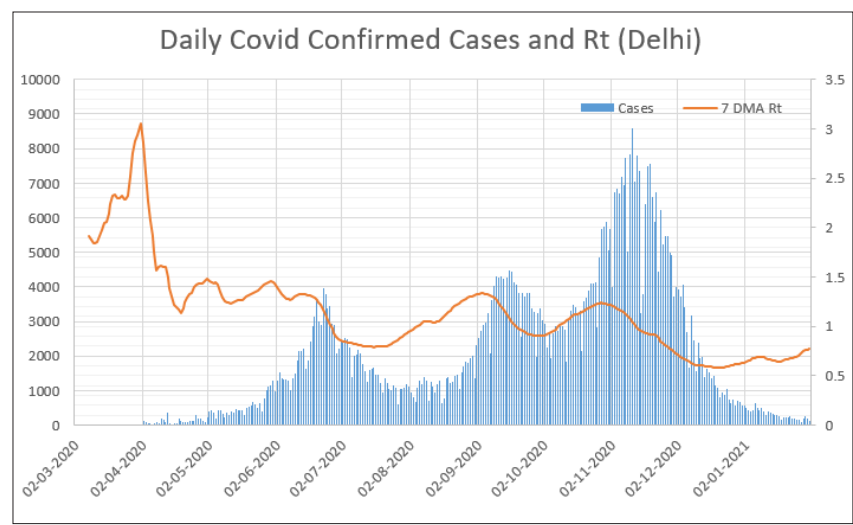

Figure 4.Daily Incidence cases of Delhi for the period (Mar 2, 2020-Jan 3 I, 202 I)

- $\quad R_{t}$ of India for February 2021 if all the NPIs are continued as usual: The value of $R_{t}$ as on Jan 31, 2021 is 0.95 with $95 \% \mathrm{Cl}(0.88,1.00)$. Since the $R_{t}$ value of India showed a declining trend, $R_{t}$ value of 0.9 was considered for the month of February in this scenario.

- $R_{t}$ of India for February 2021 if all the NPIs are removed: $R_{t}$ value of India during Pre lockdown period (March 15, 2020) was 2.4 (Figure 2). ICMR nation-wide COVID-19 sero-survey found that the prevalence of Anti-SARSCoV-2 antibodies among general population of India was $21.6 \% .{ }^{12}$ Considering the fact that the we are projecting the trajectory two months after the study and also vaccination being in place, the current $R_{t}$ value in India without NPIs was assumed to be $70 \%$ of the pre-lockdown phase, taking into account the effect of herd immunity. Hence, $R_{t}$ of 1.7 was used for the simulation model.

- $\mathrm{R}_{t}$ of Delhi for February 2021 if all the NPIs are continued as usual: The value of $R_{t}$ as on 31stJan 2021 is 0.77 with $95 \% \mathrm{Cl}[0.73,0.81]$. Since the $R_{t}$ value of Delhi showed a slowly increasing trend recently, $R_{t}$ value of 0.8 was considered for this scenario.

- $\quad R_{t}$ of Delhi for February 2021 if all the NPIs are removed: $R_{t}$ value of India during Pre lockdown period (March 15, 2020) was 2.3 (Figure 4). Previous serological surveys show a wide range of seroprevalence values. Repeated surveys have shown there is a variable trend $(23.4 \%$ in June-July, 29.1\% in August, 25.1\% in September, $25.5 \%$ in October 2020) in the serological pattern among estimates and the latest survey conducted in December found 50-60\% sero-prevalence in Delhi. ${ }^{12}$ Considering the seroprevalence data for assessing susceptible population and the fact that the $R_{t}$ value showed an upward trend during January, $R_{t}$ value in Delhi without NPIs was assumed to be $50 \%$ of the 
pre-lockdown phase. Hence, $R_{t}$ of 1.15 was used for the simulation model.

A library RO available in the statistical software R v.4.0.2 has been used for the calculation and plotting of $R_{t}$ values. The software platform used for designing the underlying model is $\mathrm{R}$ (version 4.0.2).

\section{Result}

\section{India}

Based on our model, the daily incidence of COVID-19 cases shall show an exponential rise during February 2021 if NPIs are ceased and population stop following COVID-19 appropriate behavior (wearing masks, maintaining physical distancing, hand sanitization and respiratory hygiene). This is clearly evident from the graph obtained from the simulation model as shown in (Figure 5).

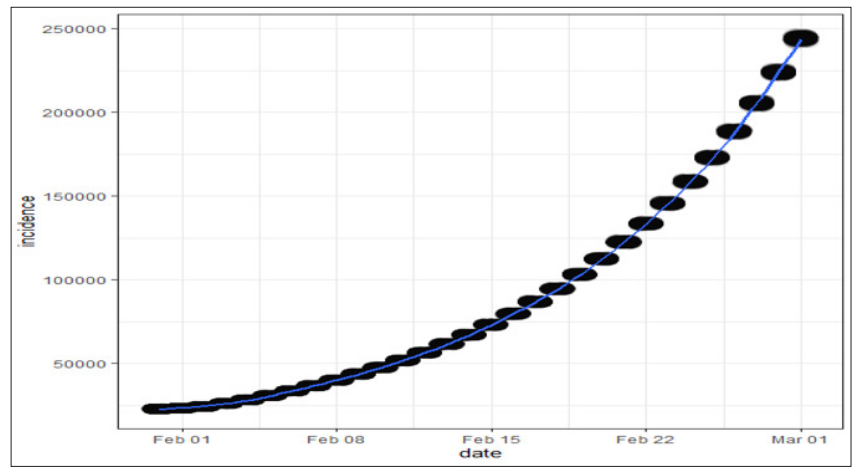

Figure 5.Future pandemic scenario of India in February with $\mathbf{R}$ value of $\mathbf{I} .7$

The expected daily incidence cases during the period for which simulation was done shows an increase from 11,500 daily cases of COVID-19 as on Jan 31, 2021 to around 75,000 daily in 15 days period and can potentially reach up to 2,50,000 daily cases by the month end/ March 1, 2021. However, in the second scenario, where all the NPIs are being implemented in the present situation are considered and it has been quantified by using $R_{t}$ value of 0.9 for India. As evident from Figure 6, the epidemic in India depicts the stable and controlled trajectory. Value of $R_{t}$ less than 1 indicates that the pandemic is in its penultimate stage indicating that it will slowly die out in the population. The cumulative incidence cases is showing a downward trend with expected cases ranging from less than 12,000 cases per day at the beginning of the month to around 8,000 cases per day by the month end, if NPIs are continued the same way as January.

\section{Delhi}

Modelling and statistical estimations from our study for Delhi too show an exponential rise in daily COVID-19 cases if NPIs are stalled. Although the rise is not as steep as that of India as a whole, but the predictions show much higher range of confidence intervals indicating high degree of variance (Figure 7 ). The actual daily incidence could be doubled in one month from 200 cases to around 400 cases daily. However, if business as usual is continued with all the existing interventional measures, daily cases can reduce to as low as 140 to 120 (Figure 8).

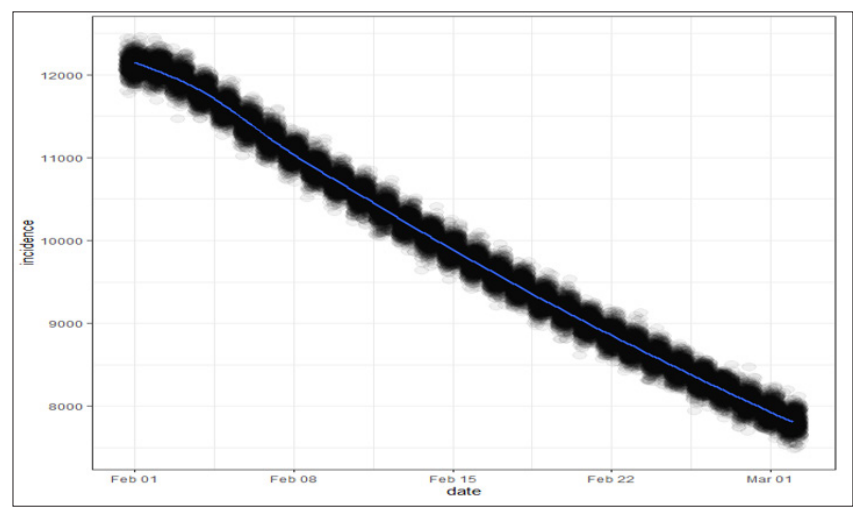

Figure 6.Future pandemic scenario of India in February with $\mathbf{R}$ value of $\mathbf{0 . 9}$

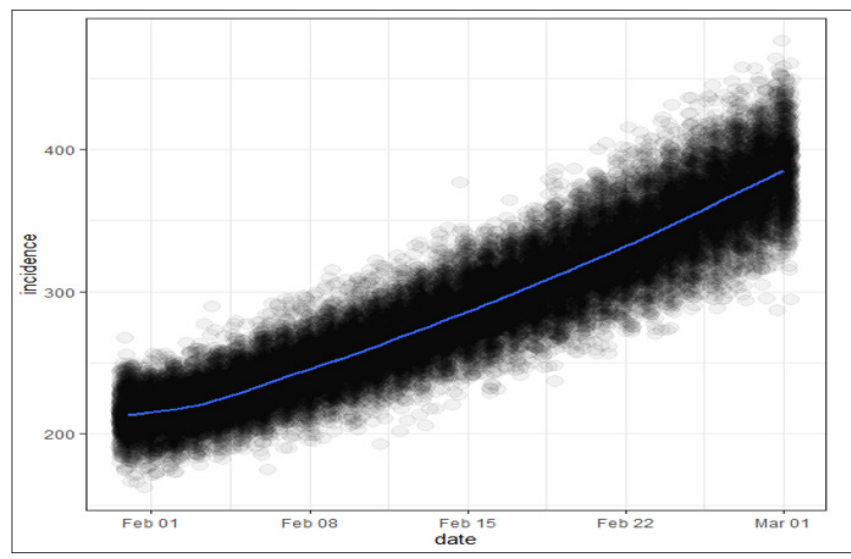

Figure 7.Future pandemic scenario of Delhi in February with $\mathbf{R}$ value of I.I5

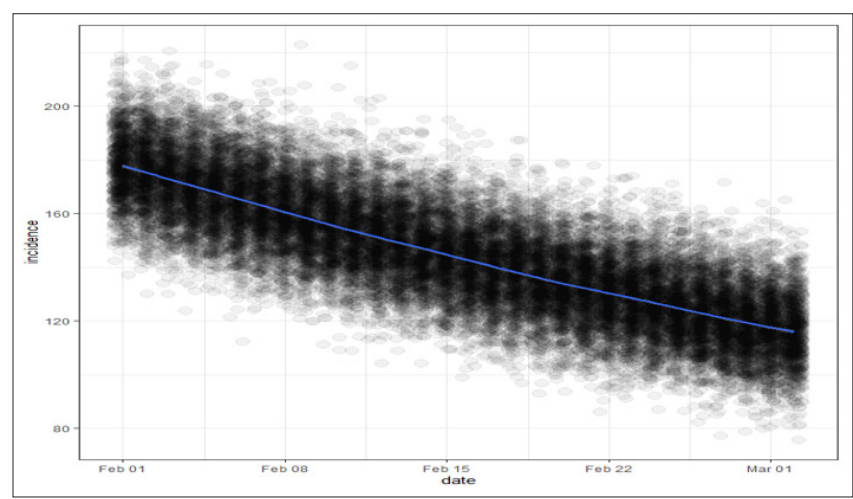

Figure 8.Future pandemic scenario of Delhi in February with $\mathbf{R}$ value of $\mathbf{0 . 8}$

\section{Discussion}

Our simulation model quantifies the effect of Nonpharmacological interventions on the current pandemic 
situation in India and Delhi, which estimated that relaxation in following preventive measures/ COVID-19 appropriate behaviors or ceasing of NPIs shall see an exponential rise in the daily incident cases. Comparing the trajectories in the Figures 5, 6 for India and Figures 7, 8 for Delhi, it can be deduced that if NPIs cease to exist for one month, the daily incident cases can get as high as 30 times normal in India and 4 times normal in Delhi by the end of February 2021. However, total cessation of NPIs is not found throughout country, therefore, rise of cases may not be as high as 30 times of normal in India. Even though vaccination program has started in India, it's necessary to understand that the effectiveness of vaccination depends on the rate of seroconversion in the general population which is yet to be tested (after 7 days of the second dose of vaccine). ${ }^{13}$ As on Feb 18, 2021, India has covered around 0.7 doses per 100 people and hence a major population is still to be covered. ${ }^{14}$ Apart from effectiveness, it's important to know the herd immunity threshold for vaccination coverage. Hopes of natural herd immunity at seroprevalence level as high as $66 \%$ estimate after the $1^{\text {st }}$ wave of COVID-19 in Manaus, Brazil has resulted in disappointment due to resurgence of the virus observed as a second wave of COVID-19 cases. ${ }^{15-17}$ Such instances have raised questions about the extent vaccination coverage needed for obtaining herd immunity status. There are also evidences indicating duration of anti-SARS-CoV-2 antibodies last much shorter period in India. ${ }^{18}$ Studies on duration of antibody presence in post seroconversion phase of vaccination through longitudinal follow-up studies are also essential to be sure about the effectiveness of the vaccination program. Hence, all these factors suggest that even during the phase of post vaccine roll-out, NPIs remain to play a major role in containing the spread and minimizing the effects of the COVID-19 pandemic. Any kind of relaxation in NPIs can lead to sudden surge of incident cases and correspondingly may increase the death toll. Hence, a graded approach in getting back to normalcy has to be continued. As it has been more than a year since COVID-19 was declared Public health emergency of international concern, there is a high possibility that there could be reluctancy among general population in following COVID-9 appropriate behaviors, amplified by the inappropriate overestimation of impact from vaccination at the current stage. It is recommended to continue or accelerate the Risk Communication and Community Engagement (RCCE) strategies for the best benefit.

\section{Limitation of the Model}

Scenario is generated for the small period of time since projecting the values for longer period may compromise the reliability of the estimates. Also, a constant $R_{t}$ value has been assumed for the simulated period assuming the most probable average for the month even though it is evident that daily $R_{t}$ value varies as per the daily incidence cases.

\section{Conflict of Interest: None}

\section{References}

1. https://www.who.int/emergencies/diseases/ novel-coronavirus-2019/interactive-timeline? gclid=CjwKCAiA57D_BRAZEiwAZcfCxbWNckPtix V5WMvb8G1wyg1M-iX2ih8Bm0_pY6NiKDafM yciu6x5YRoCaroQAvD_BwE\#

2. Andrews MA, Areekal B, Rajesh KR et al. First confirmed case of COVID-19 infection in India: A case report. The Indian Journal of Medical Research 2020; 151(5): 490.

3. https://www.mohfw.gov.in/

4. Ministry of Health and Family Welfare. COVID-19 Vaccine Rollout. 16 JAN 2021 9:05PM by PIB Delhi. Accessed Feb 10, 2021. Available from:https://pib.gov. in/PressReleasePage. aspx?PRID $=1689180$

5. Ministry of Health and Family Welfare. COVID-19 Vaccine Rollout. 17 JAN 2021 9:05PM by PIB Delhi. Accessed Feb 10, 2021. Available from:https://pib.gov. in/PressReleasePage.aspx?PRID=1689167

6. MoHFW. Frequently Asked Questions. Accessed Feb 10, 2021. Available from:https://www.mohfw.gov.in/ covid_vaccination/vaccination/index.html

7. https://www.indiatvnews.com/news/india/lockdownunlock-in-india-covid19-pandemic-guidelinesrestrictions-2020-coronavirus-lockdown-series-674925

8. https://www.repidemicsconsortium.org/projections/

9. Patel P, Athotra A, Vaisakh TP et al. NCDC COVID Incident Management Team. Impact of nonpharmacological interventions on COVID-19 transmission dynamics in India. Indian J Public Health 2020; 64: S142-6.

10. https://www.covid19india.org/

11. https://www.bbc.com/news/world-asiaindia-55945382

12. https://scroll.in/latest/985948/21-5-of-indiaspopulation-has-past-exposure-to-coronavirus-showsicmrs-third-sero-survey

13. https://ourworldindata.org/grapher/covid-vaccinationdoses-per-capita?tab=chart\&stackMode=absolute \&c ountry $=\sim$ IND\&region=World

14. https://ourworldindata.org/grapher/covid-vaccinationdoses-per-capita?tab=chart\&stackMode=absolute $\& c$ ountry $=\sim$ IND\&region=World

15. Buss LF, Prete CA, Abrahim CM et al. COVID-19 Herd Immunity in the Brazilian Amazon. Infectious Diseases (except HIV/AIDS); 2020. doi:10.1101/ 2020.09.16.20194787

16. Signorelli C, Zucchi A, Tersalvi CA et al. High seroprevalence of SARS_COV-2 in Bergamo: evidence for herd immunity or reason to be cautious? Int J 
Public Health 2020; 65(9): 1815-1817. DOI:10.1007/ s00038-020-01524-x

17. The Scientist. News article. A study estimates that $76 \%$ of the population of the city of Manaus in Brazil has been exposed to Covid. Dec 14, 2020. Available from: https://www.the-scientist.com/news-opinion/studyestimates-76-percent-of-brazilian-city-exposed-tosars-cov-2-68272

18. Kumar N, Bhartiya S, Singh T. Duration of anti-SARSCoV-2 antibodies much shorter in India. Vaccine 2020 Nov. DOI: 10.1016/j.vaccine.2020.10.094. 